\title{
Relativity at the International Congress of Philosophy, Naples.
}

SINCE the inception of the International Congresses of Philosophy in I900, it was arranged that such gatherings should be an opportunity for philosophers and men of science to meet on common ground for discussion. Nowadays, philosophers cannot claim to have a complete system unless they account for the various branches of science in their synthetic explanation of things. They must understand what men of science have found out for themselves and give a value to their discoveries in their theories of knowledge. It is quite appropriate then that they should be helped in their efforts by the searching analysis scientific workers have made of their own labours. There cannot be any antagonism between philosophy and science; one completes the other and each is strengthened by the support of the other.

It was a gratifying spectacle to see metaphysicians, logicians, mathematicians, physicists, and biologists sitting together in the great halls of the University of Naples. These meetings formed an integral part of the International Congress of Philosophy, which was arranged at Naples at the beginning of May, under the presidency of Prof. Aliotta, on the occasion of the seventh centenary of the foundation of that University.

Unavoidably the outstanding question under discussion during these joint meetings was Einstein's theory of relativity. Prof. Einstein was, however, prevented from attending the Congress at the last moment. Nevertheless, some valuable papers were read at the sittings of the section of History and Philosophy of Science. Under the chairmanship of Prof. Marcolongo of Naples and then of Prof. Hadamard of Paris, various aspects of the theory of relativity were developed. Prof. Cartan (Paris), in a paper on "The Theory of Relativity and the Generalisation of the Notion of Space," gave an interpretation of Einstein's tensor by means of the notion of curvature and that of the torsion of a curved space, using also Levi-Civita's definition of parallelism. Prof. Hadamard put forward some interesting considerations on the equations of cylindric waves already studied by Volterra. $M$. Nordmann, of the National Observatory of Paris, dealt with the controversy between Einstein and Bergson with reference to the relativity of time. He contended that Einstein's memoir, published in 1905, gives no ground for criticism, and that Bergson's remarks are based on certain statements, made by Einstein in his popular work, which are open to controversy.

Mr. Zaremba of Cracow, in a paper, "The Theory of Relativity and Experience," pointed out some logical failures of Einstein's theory, and expressed the opinion that at the present stage it cannot be said that experience is for or against it. Prof. Kopff (Berlin) developed some suggestive relations between astronomy and Einstein's conceptions.

Prof. Enriques (Rome), in a paper entitled " A Criticism of the Notion of Time," maintained that time is not only, as according to Kant, the order of internal sensibility, but also that it contains the idea of a natural standard of measure. The analysis of the postulates referring to such a standard reveals the unity of physical forces and the hypothesis that time is independent of space. The rejection of this hypothesis leads to the theory of relativity. Prof. Giorgi acknowledged that the mathematical and logical structure of Einstein's theory is absolutely perfect and quite compatible with natural phenomena. But he does not think that this theory has had sufficient verifications in proportion to its importance and ambitions. Prof. Severi expounded a new method of arriving at Lorentz's classical formulæ by means of an analysis of the concept of time. Prof. Timpanaro, speaking on "The Value of the Theory of Relativity," proposed a new theory, which he called etheral-baliistic, according to which natural phenomena can be explained with as much exactness as with the theory of relativity.

The great opponent of Einstein's ideas was Prof. La Rosa, of Palermo, who spoke on "Some Astronomical Facts against Einstein's Theory based on Ritz's Hypothesis." He showed how to explain several phenomena of variable stars according to Ritz's hypothesis where the velocity of light is compounded with that of force. Einstein's theory, he said, is only one of the possible relativistic ways of interpreting natural phenomena. There is, besides, what he called the "ballistic method," which has much experimental evidence in its favour. The ballistic hypothesis states that the velocity of light can be compounded with that of its source; and when applied to the theory of the double stars it leads to some very interesting and unexpected results. For example, on the basis of the ballistic hypothesis, all double stars having a distance and a period satisfying certain limitations, must appear to us as variable stars. Following up this line of thought, Prof. La Rosa offers a general explanation of the phenomena of double and variable stars.

Among other papers on the philosophy of science, we must mention those of Prof. Vorovka (Prague) and Sittignani, who discussed from different angles Poincaré's epistemological theories, and Prof. Driesch's communication on " The Philosophy of Organic Life." Prof. Hans Driesch, the most authoritative representative of vitalism, explained, with an abundance of examples, that the phenomena of life cannot be accounted for simply by means of physico-chemical processes. Biology shows us that a multicellular organism can be divided up into many parts without stopping its development. A machine cannot be submitted to the same operation with the same results. There must be, therefore, in every living organism a cohesive agent, which he calls "entelechy," the function of which cannot be analysed at the present stage of science, but obviously it causes the organism to develop whatever be the mishaps it may suffer.

In his paper on " The Specification of the Straight Line," Dr. T. Greenwood developed the logical arguments which lead him to state in a new way the problem of the characterisation of the Euclidian straight line by means of a single axiom which he calls "The Postulate of Null-Curvature." He establishes this postulate by means of a new hypothetico-deductive system of axioms based on the notions of "point" and "distance," and uses it to prove the two ordinary postulates of the straight line. This method, which is very simple in itself, has many advantages in the logical, the pedagogical, and the scientific field.

The numerous papers, more than two hundred, read at the Naples Congress did not permit of any discussion, mainly because the time available was not proportionate to their number. This, however, shows admirably the passionate devotion with which. Italian thinkers study science and philosophy; and the more so as, besides the International Congress of Philosophy, an Ophthalmological Congress, a Gynæcological Congress, and a joint meeting of the Association of Scientific Societies of Italy, were sitting concurrently at Naples, whilst there was a Sociological Congress and a Eugenics Congress at Rome, and a Geographical Congress at Genoa. Yet, to all those who were able to go to Naples-unfortunately there were not many visitors from Great Britain - the visit will rank among the most pleasant memories of their academical career.
Thomas GREENWOOD. 